\title{
Improved Time Domain BRS Assessment with the Use of Baroreflex Events
}

\author{
S Gouveia ${ }^{1,2}$, AP Rocha ${ }^{1,2}$, P Laguna $^{3}$, P Lago $^{1}$ \\ ${ }^{1}$ Dep de Matemática Aplicada, Faculdade de Ciências, Universidade do Porto, Portugal \\ ${ }^{2}$ Centro de Matemática da Universidade do Porto, Portugal \\ ${ }^{3}$ Comm Tech Group, Aragon Institute of Eng Research / CIBER-BBN, Univ of Zaragoza, Spain
}

\begin{abstract}
The sequences technique is based on a linear regression of $S B P$ and $R R$ values in baroreflex sequences (BSs). In normal conditions most of BSs have 3 beats length and the estimated slope is potentially inaccurate. Also, the number of beats in BSs is approximately $25 \%$ of the total number of beats, evidencing that a large part of the data is discarded from BRS analysis.

In this work, global BRS estimators combined with baroreflex events (BEs) are proposed as a way to improve BRS assessment in normal conditions and to allow its quantification in cases of BS absence.

The results in the EuroBaVar dataset showed that the number of beats in BEs gets up to 50\% of the total, with correlation between the corresponding $S B P$ and RR values close to 0.8. Also, BRS assessment based on global estimators combined with BEs distinguishes Lying from Standing positions in all the subjects of that dataset, including those without identified BSs.
\end{abstract}

\section{Introduction}

The sequences technique is the most used time domain method for arterial baroreflex sensitivity (BRS) assessment. It is based on a linear regression over the systolic blood pressure (SBP) and RR interval values, identified in each baroreflex sequence (BS) [1]. An overall BRS estimate is usually obtained from the mean of the slopes estimated at each BS (local approach). Previous work described alternative global estimators, with BRS being estimated as the slope of all SBP and RR values in the set of all BSs [2,3]. The results showed that the local and global estimated values are highly correlated and that global estimates present less bias, less variance and superior ability to distinguish lying from standing positions.

In normal conditions more than $50 \%$ of the BSs have only 3 beats length and the slope estimated at each BS is potentially inaccurate. Also, the overall number of beats in BSs is around $25 \%$ of the total and a large part of data is discarded from BRS analysis. In cases of ANS dysfunc- tion, the lack of data can be even more problematic, because the number of BSs is further reduced or completely unavailable. If unavailable, BRS can not be quantified. If in a reduced number, the slope estimation will be based on few data and will present increased variance. Therefore, the validity of the BRS values estimated with short and few segments of SBP/RR data needs to be addressed.

In this work, BRS estimation from systolic ramps (SRs) [3] and from baroreflex events (BEs) are discussed as alternatives to the traditional BRS estimation from BSs.

Figure 1 shows SBP/RR segments in identified BSs, SRs and BEs. It can be seen that SRs are longer segments than BSs and that the beats identified in BSs are also identified in SRs, but not vice versa [3]. Also, BEs are much longer segments than SRs and BSs. Therefore, BRS analysis combined with BEs can benefit from the higher number of beats for slope estimation.

(a)

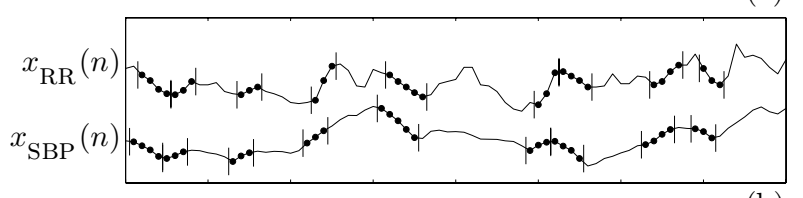

(b)

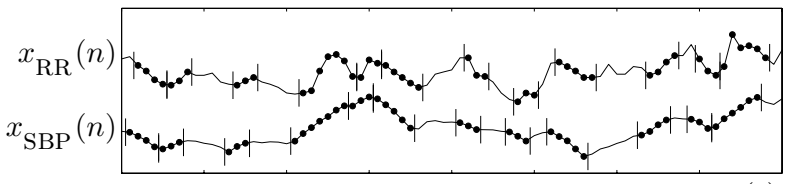

(c)

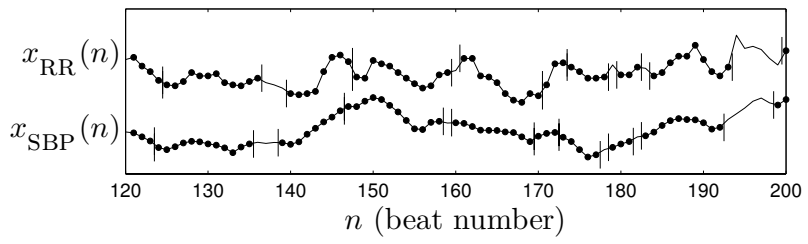

Figure 1. Segments of SBP and RR values in baroreflex sequences or BSs (a), systolic ramps or SRs (b) and baroreflex events or BEs (c), identified in the "A001LB" file of the EuroBaVar dataset [4]. 


\section{Methods}

Time domain methods for BRS assessment are based on the identification of baroreflex related segments ( $\sec 2.1$ ) and on the BRS estimation as the linear slope between the corresponding SBP and RR values ( $\sec 2.2$ ).

The BRS analysis is performed over SBP and RR series of 512 beats length with 1 beat lag, i.e., each SBP value is paired with the subsequent RR interval [1].

\subsection{Identification of baroreflex segments}

The BRS analysis is performed over the SBP and RR series, respectively denoted as $x_{\mathrm{SBP}}$ and $x_{\mathrm{RR}}$. The values of the lower thresholds required for the identification of valid BSs, SRs and BEs are summarized in Table 1.

Each valid $\mathrm{BS}_{k}, k=1,2, \ldots, K$ requires a minimum length in beats $\left(N_{k} \geq N_{\text {min }}\right)$, minimum $x_{\mathrm{SBP}}$ and $x_{\mathrm{RR}}$ beatto-beat changes in the same direction $\left(\Delta_{k}^{\mathrm{SBP}} \geq \Delta_{m i n}^{\mathrm{SBP}}\right.$ and $\Delta_{k}^{\mathrm{RR}} \geq \Delta_{\text {min }}^{\mathrm{RR}}$ ) and a minimum value of $r_{\text {min }}$ for the correlation between the $x_{\mathrm{SBP}}$ and $x_{\mathrm{RR}}$ values in BSs.

For each $k^{t h}$ valid systolic ramp $\mathrm{SR}_{k}$, only $N_{\text {min }}$ and $\Delta_{\text {min }}^{\mathrm{SBP}}$ are imposed, regardless of the possible occurrence of concomitant changes in $x_{\mathrm{RR}}$.

The criterion for SBP-RR segmentation proposed in this work, consists in the identification of segments that exhibit high correlation between the corresponding $x_{\mathrm{SBP}}$ and $x_{\mathrm{RR}}$ values. For each $k^{\text {th }}$ valid baroreflex event $\mathrm{BE}_{k}$ only the thresholds $N_{\min }$ and $r_{\min }$ are enforced. Since no minimum values for $\Delta_{k}^{\mathrm{SBP}}$ and $\Delta_{k}^{\mathrm{RR}}$ are required, $x_{\mathrm{SBP}}$ and $x_{\mathrm{RR}}$ might not be consecutively increasing or decreasing as in BSs, as long as the correlation between $x_{\mathrm{SBP}}$ and $x_{\mathrm{RR}}$ values is positive and higher than the minimum threshold $r_{\text {min }}$. Under the best of conditions, if the $x_{\mathrm{SBP}}-x_{\mathrm{RR}}$ correlation found in one recording is higher than $r_{m i n}$, all beats are considered for BRS analysis with BEs.

Table 1. Lower thresholds for BS/SR/BE identification.

\begin{tabular}{|c||c|c|c||c|}
\hline Threshold & BS & SR & BE & Units \\
\hline$N_{\text {min }}$ & 3 & 3 & 3 & beats \\
$\Delta_{\text {min }}^{\mathrm{SBP}}$ & 1 & 1 & - & $\mathrm{mmHg}$ \\
$\Delta_{\min }^{\mathrm{RR}}$ & 5 & - & - & $\mathrm{ms}$ \\
$r_{\min }$ & 0.8 & - & 0.8 & - \\
\hline
\end{tabular}

\subsection{BRS estimation}

BRS estimates can be based on local, global or total approaches combined with BSs, SRs or BEs. The local approach provides a BRS measure associated to each BS [1] and the mean of the local slopes, $\mathcal{B}_{\mathrm{L}, \mathrm{O}}$, is used as an overall estimator. Alternatively, a global BRS measure can be considered [2], summarized as follows. After segments identification, the mean is removed from $x_{\mathrm{SBP}}$ and $x_{\mathrm{RR}}$ at each segment. The SBP and RR detrended values are then concatenated in $\mathbf{d}_{\mathrm{SBP}}$ and $\mathbf{d}_{\mathrm{RR}}$ vectors, respectively. Finally, the global slope $\mathcal{B}_{\mathrm{G}, \mathrm{O}}$ is obtained from

$$
\mathbf{d}_{\mathrm{RR}}=\mathcal{B}_{\mathrm{G}, \mathrm{O}} \mathbf{d}_{\mathrm{SBP}}+\boldsymbol{\epsilon},
$$

where the parameter $\mathcal{B}_{\mathrm{G}, \mathrm{O}}$ is estimated by ordinary least squares (OLS) minimization and $\epsilon$ is a vector of residuals.

The total approach based on $\mathcal{B}_{\mathrm{G}, \mathrm{T}}$ estimator constitutes a more robust global approach, that combines an outlier rejection rule with slope estimation by total least squares (TLS) minimization [3]. In linear regression the OLS method attributes all errors to the $d_{R R}$ variable in equation (1), whereas the TLS method minimizes the sum of the squared orthogonal errors, accounting for errors in both the $\mathbf{d}_{\mathrm{SBP}}$ and $\mathbf{d}_{\mathrm{RR}}$ variables. Before TLS slope estimation, outlier segments are removed from BRS analysis, as follows. The influence of the $k^{t h}$ segment is evaluated by the ratio $f_{k}=\left(T L S\right.$ slope when the $k^{\text {th }}$ segment is ommited from the analysis)/(TLS slope when all data is used). Baroreflex segments are considered as outliers if their influence differ from the median of influences more than two median absolute deviations (MAD) divided by 0.6745 [3]. As illustrated in Figure 2, the rejection criterion allows the identification of BSs, SRs and BEs with unusual high influence on the slope estimation. Also, the narrower $95 \%$ robust acceptance band for BEs indicates greater homogeneity between the BEs influences.
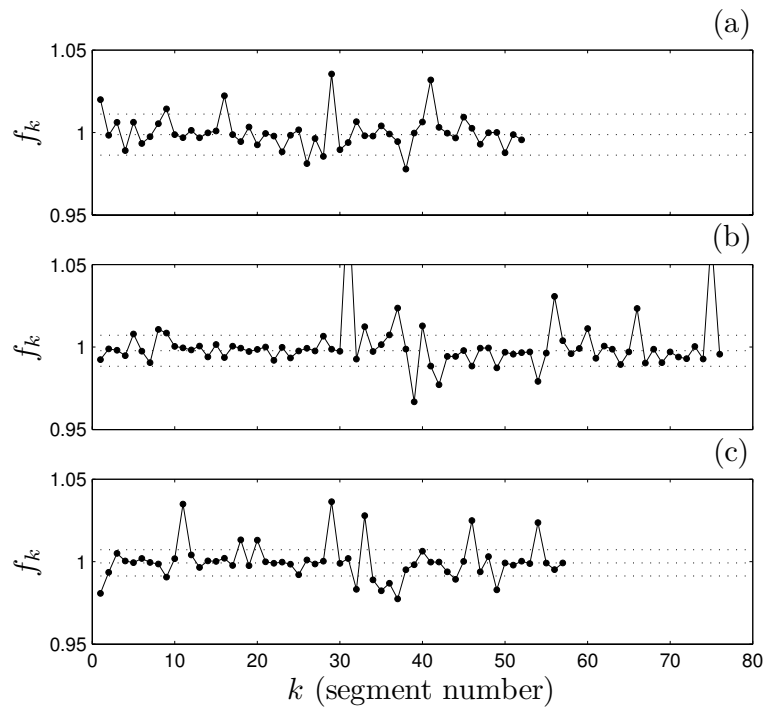

Figure 2. Influence function for the identified BS/SR/BE $(\mathrm{a} / \mathrm{b} / \mathrm{c})$, with the $95 \%$ robust acceptance band. Same data as in Figure 1. 
After outlier removal the TLS slope $\alpha$ is estimated with the remaining pairs of values $\left(\mathbf{d}_{\mathrm{SBP}, \alpha}, \mathbf{d}_{\mathrm{RR}, \alpha}\right)$, divided by the corresponding MAD values. This normalization compensates the inherent dependence of the TLS method on scale changes and guarantees the same order of magnitude in the variables. Finally, $\mathcal{B}_{\mathrm{G}, \mathrm{T}}$ is obtained from

$$
\mathcal{B}_{\mathrm{G}, \mathrm{T}}=\frac{\operatorname{MAD}\left(\mathbf{d}_{\mathrm{RR}, \alpha}\right)}{\operatorname{MAD}\left(\mathbf{d}_{\mathrm{SBP}, \alpha}\right)} \alpha .
$$

Figure 3 summarizes the BRS estimation results using local/global/total approaches combined with BS/SR/BE. Figures 3(a) evidence that $N$ is higher in BEs. Also, the corresponding $\mathbf{d}_{\mathrm{SBP}}$ and $\mathbf{d}_{\mathrm{RR}}$ values exhibit more variability than in BSs and their linear relation is more evident than in SRs. Figures 3(b) illustrate the data after outlier removal. The extreme points of the outlier segments are out of the mass center of the data and the $\hat{\mathcal{B}}_{\mathrm{G}, \mathrm{T}}$ slope is more in accordance with the global relationship of the data.

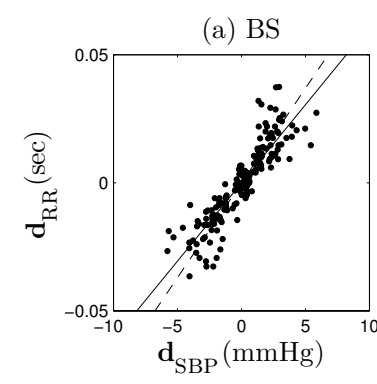

(a) SR

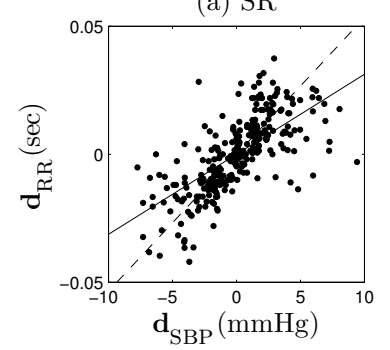

(a) $\mathrm{BE}$

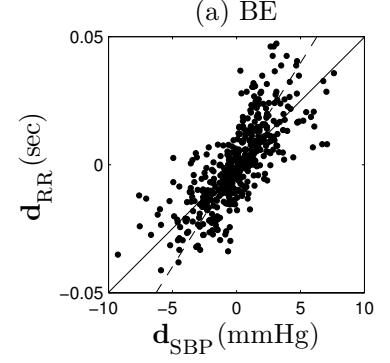

(b) BS

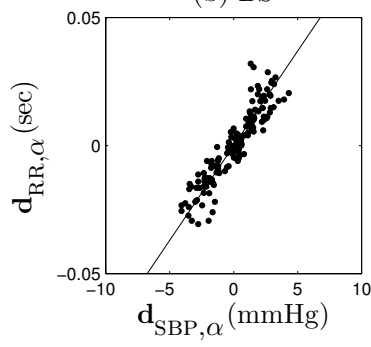

(b) SR

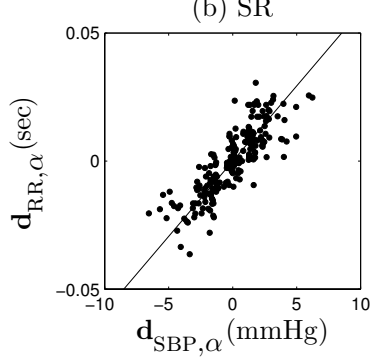

(b) $\mathrm{BE}$

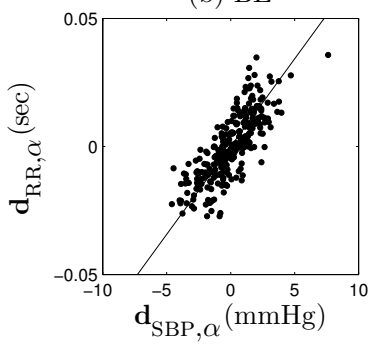

Figure 3. (a): $\mathbf{d}_{\mathrm{SBP}}$ and $\mathbf{d}_{\mathrm{RR}}$ values superimposing the dashed line with slope $\hat{\mathcal{B}}_{\mathrm{L}, \mathrm{O}}$ passing through the origin and the line with slope $\hat{\mathcal{B}}_{\mathrm{G}, \mathrm{O}}$. (b): $\mathbf{d}_{\mathrm{SBP}, \alpha}$ and $\mathbf{d}_{\mathrm{RR}, \alpha}$ values and the line with slope $\hat{\mathcal{B}}_{\mathrm{G}, \mathrm{T}}$. Values identified in BS/SR/BE. Same data as in Figure 1.

\section{Results}

The application of the methods is illustrated with the EuroBaVar dataset [4], composed of 46 records with SBP and RR series, paired in lying $(L)$ or standing $(S)$ positions. The methods are compared regarding the number of beats for BRS analysis $(N)$, the number of identified segments $(K)$, the $\mathbf{d}_{\mathrm{SBP}}-\mathbf{d}_{\mathrm{RR}}$ correlation $(r)$ and the ability to discriminate $L$ from $S$ for each record.

Figures 4(a)-(f) evidence that BRS analysis with the use of BEs is advantageous. Comparing with BSs, BEs present

- higher $N$,

- lower $r$, although close to 0.8 and

- higher $K$ and

- higher length.

In comparison with SRs, BEs present

- similar $N$ and larger interindividual differences,

- higher $r$,

- lower $K$ and

- higher length.

Figure $4(\mathrm{~g})$ illustrates the distribution of $\hat{\mathcal{B}}$. With the use of BSs, $\hat{\mathcal{B}}$ shows large interindividual differences. The values of $\hat{\mathcal{B}}$ estimated from SRs are the lowest and also present less variability. In contrast, $\hat{\mathcal{B}}$ estimated from BEs are the highest and show the largest interindividual differences. With the use of the global/total approach, $\hat{\mathcal{B}}$ estimated from BSs and BEs have similar distributions.

For $L$ and $S$ discrimination, it is expected that the $L$ to $S$ ratio of the $\hat{\mathcal{B}}$ values to be above $1\left(\hat{R}_{L S}>1\right)$ [4]. As illustrated in Figure 4(h), there is strong evidence that both $\hat{R}_{L S}$ mean and median are above 1 , for all approaches combined with BSs or BEs. Of the 23 cases, 21 present BSs in both positions and the $\hat{R}_{L S}$ values estimated by local/global/total approach are above 1 in 18/20/20 of the pairs. All 23 pairs present both SRs and BEs, and $\hat{R}_{L S}$ are above 1 in 15/18/15 for SRs and in 20/23/23 of the pairs for BEs. The use of global/total approaches combined with BEs distinguish $L$ from $S$ in all cases of the EuroBaVar dataset, including those without BSs.

The circles in Figures 4 correspond to the four files without identified BSs (B005 and B010 both for $L$ and $S$ positions). The BRS estimation from SRs and BEs has the capability of providing an estimate even when BSs are not identified. The values of $N$ and $K$ evaluated in SRs and in BEs do not correspond to the lowest values found in the dataset. The corresponding $\hat{\mathcal{B}}$ obtained from SRs are low (and even negative). However, as $r<0.3$ for these files, the small values of $\hat{\mathcal{B}}$ could be a consequence of the poor fit of the linear model and may not be associated with the physiological conditions of the subjects. With the use of BEs, low $\hat{\mathcal{B}}$ values are also estimated with corresponding $r$ values similar to $r$ of the remaining files. All approaches combined with BEs distinguish $L$ from $S$ also for the files without BSs. 


\section{Conclusions}

In this work the use of the baroreflex sequences (BSs) and baroreflex events (BEs) for time domain BRS analysis is discussed. With the use of BEs instead of BSs, there are more beats for slope estimation, because BEs are longer segments and/or in higher numbers (in median, around $50 \%$ of all beats are in BEs against $25 \%$ in BSs). The correlation between the corresponding SBP and RR values in BEs is lower than in BSs, but still close to 0.75. In cases of BSs absence (usually associated with BRS failure), low BRS values are estimated from BEs. The global/total estimates with the use of BEs are able to discriminate $L$ from $S$ positions, even in the cases where no BSs are identified. Superior results are obtained with the total approach, considering the median values. Therefore, in more diversified situations corresponding to clinical practice, it is expected that the robust properties underlying the total approach can be fully enhanced.

In short, the global/total approaches combined with BEs improve time domain BRS assessment, leading to a higher number of beats for slope estimation, a superior ability to discriminate lying from standing positions and the capability of providing an adequate BRS estimate, even in subjects where BSs are not identified.

\section{Acknowledgements}

This work was partially supported by CMUP (financed by FCT Portugal through POCI2010/POCTI/POSI programmes, with national and CSF funds) and TEC200405263-C02-02 from CICYT/FEDER Spain. S Gouveia acknowledges the grant SFRH/BD/18894/2004 by FCT/ESF.

\section{References}

[1] Bertineri G, Di Rienzo M, Cavallazzi A. Evaluation of baroreceptor reflex by blood pressure monitoring in unanesthetized cats. Am J Physiol 1988;254:H377-383.

[2] Gouveia S, Rocha AP, Borne P, Lago P. Assessing baroreflex sensitivity in the sequences technique: local versus global approach. Proc Comput Cardiol 2005;32:279-282.

[3] Gouveia S, Rocha AP, Laguna P, Borne P, Lago P. Improved BRS assessment using the global approach in the sequences technique. Proc Comput Cardiol 2006;33:641-644.

[4] Laude D et al. Comparison of various techniques used to estimate spontaneous baroreflex sensitivity. Am J Physiol Regul Integr Comp Physiol 2004;286(1):R226-231.

\section{Address for correspondence:}

\section{Sónia Gouveia}

Dep Matemática Aplicada, Faculdade Ciências Univ Porto

Rua do Campo Alegre, 687; 4169-007, Porto, Portugal.

E-mail address: sagouvei@fc.up.pt. (a)

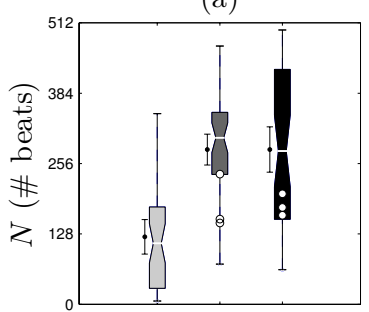

(c)

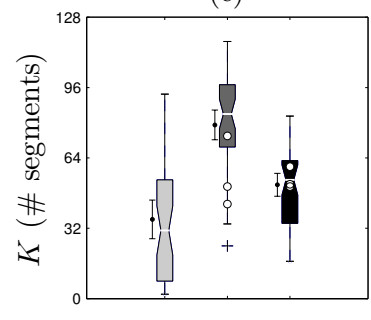

(e)
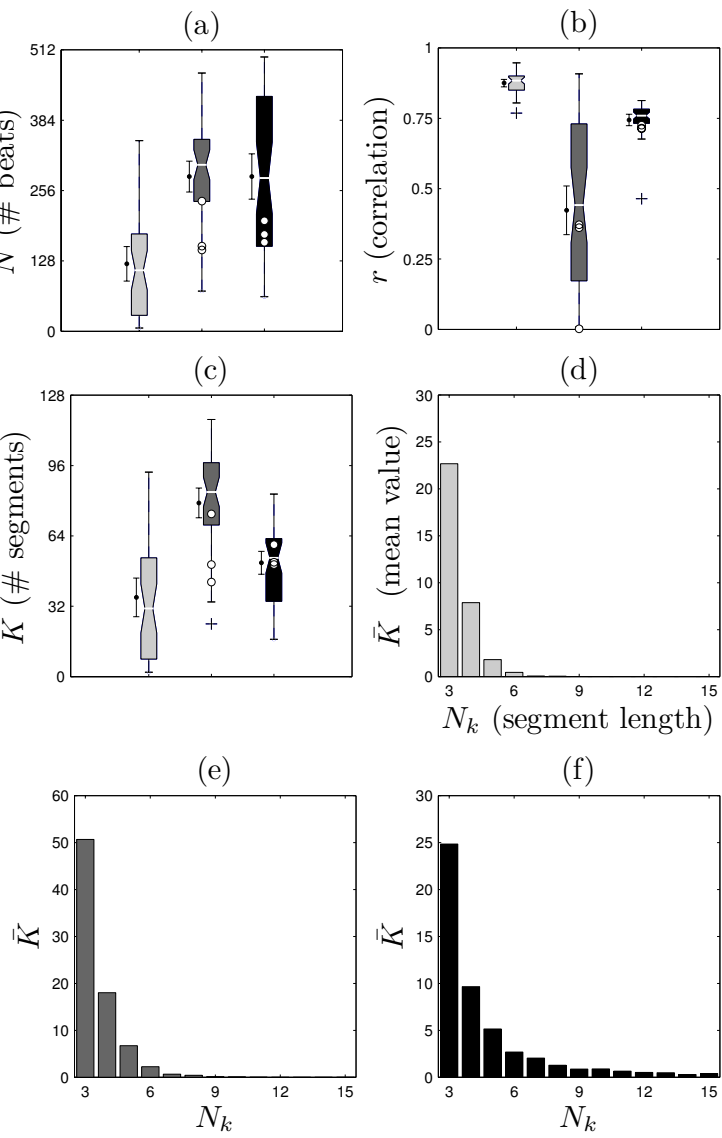

(d)

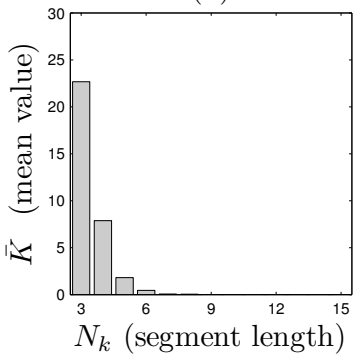

(f)
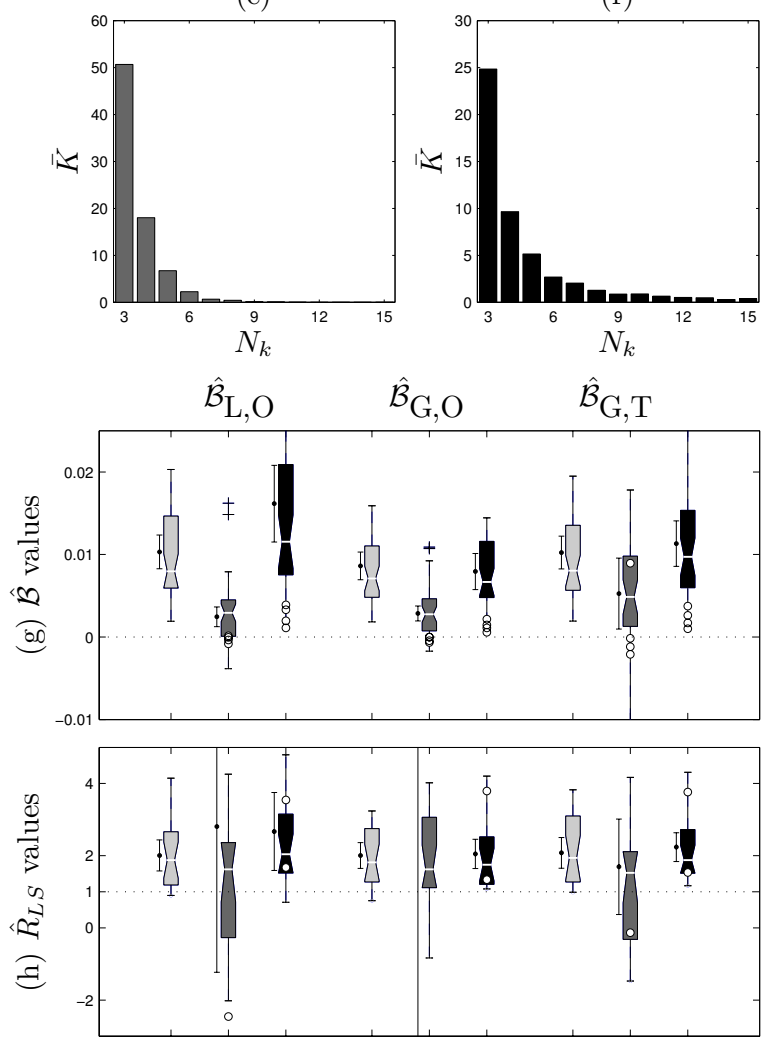

Figure 4. (a)-(c): Boxplot of $N, r$ and $K$. (d)-(f) Distribution of the mean number of segments in each file $(\bar{K})$ per segments length $N_{k}$. Boxplot of (g) $\hat{\mathcal{B}}$ and (h) $L$ to $S \hat{\mathcal{B}}$ ratio. Outliers not represented in the axis. Median and mean $95 \%$ confidence intervals represented by the notch and by the interval at the left of each boxplot, respectively. Values estimated from BS/SR/BE $(\diamond / \bullet / \bullet)$. The circles place the values obtained for the files without BS. 